\title{
Autoimmune vitiligo in rheumatic disease in the mestizo Mexican population
}

\author{
ESPERANZA AVALOS-DÍAZ* , ELENA PÉREZ-PÉREZ* , MAYRA RODRÍGUEZ-RODRÍGUEZ, \\ MARÍA-GUADALUPE PACHECO-TOVAR and RAFAEL HERRERA-ESPARZA
}

Department of Immunology, UACB, Universidad Autónoma de Zacatecas, Zacatecas 98040, Mexico

Received March 9, 2016; Accepted June 1, 2016

DOI: $10.3892 / \mathrm{br} .2016 .700$

\begin{abstract}
Vitiligo is a chronic disease characterized by the dysfunction or destruction of melanocytes with secondary depigmentation. The aim of the present study was to determine the prevalence of vitiligo associated with autoimmune rheumatic diseases. The clinical records from a 10-year database of patients with rheumatic diseases and associated vitiligo was analysed, with one group of patients having autoimmune rheumatic disease and another non-autoimmune rheumatic disease. Available serum samples were used to assess the anti-melanocyte antibodies. A total of 5,251 individual clinical files were archived in the last 10 years, and these patients underwent multiple rheumatology consultations, with $0.3 \%$ of the group presenting with vitiligo. The prevalence of vitiligo in the autoimmune rheumatic disease group was $0.672 \%$, which was mainly associated with lupus and arthritis. However, patients with more than one autoimmune disease had an increased relative risk to develop vitiligo, and anti-melanocyte antibodies were positive in $92 \%$ of these patients. By contrast, the prevalence was $0.082 \%$ in the group that lacked autoimmune rheumatic disease and had negative autoantibodies. In conclusion, the association between vitiligo and autoimmune rheumatic diseases was relatively low. However, the relative risk increased when there were other autoimmune comorbidities, such as thyroiditis or celiac disease. Therefore, the presence of multiple autoimmune syndromes should be suspected.
\end{abstract}

\section{Introduction}

Vitiligo is a skin disease characterized by dysfunction or destruction of melanocytes with secondary depigmentation,

Correspondence to: Dr Esperanza Avalos-Díaz, Department of Immunology, UACB, Universidad Autónoma de Zacatecas, Chepinque 306, Colonia Lomas de la Soledad, Zacatecas 98040, Mexico

E-mail: avalosespera@gmail.com

*Contributed equally

Key words: vitiligo, autoimmunity, rheumatic disease which constitutes the pathophysiological hallmark of the disease. The pathogenesis of vitiligo involves multiple factors. In 1950, Lerner proposed the neural theory (1). Since then, different evolving theories for the pathogenesis of vitiligo have been mentioned, including susceptibility genes, autoimmune response, abnormalities in the melanocytes and/or cellular damage from oxidative stress.

Genes for generalized vitiligo $(\mathrm{GV})$ include $X B P 1, F O X P 3$, $N A L P q, T Y R$, and TLSP (2). Additionally, certain MHC genes are also associated with vitiligo, including the $H L A-D R B 1^{*} 07$, $H L A-A 2$ and $H L A-B 17$ alleles. Similarly, SNAPs genotyping studies have demonstrated that the $6 \mathrm{q} 27$ chromosome is linked to this skin depigmentation disorder (3). The pathophysiology of autoimmune vitiligo involves humoral and cellular immunity by mechanisms that produce melanocyte damage. Clinically, depigmentation can be a unique symptom associated with autoantibody production and/or cell hypersensitivity. However, in a few cases of GV, the skin disease can be associated with other autoimmune diseases. With respect to the mechanisms involved in the autoimmune destruction of melanocytes, CD8 T cells appear to trigger melanocyte damage. Then, vitiligo antigens are released by cytolysis. Therefore, the initial insult is followed by a humoral autoimmune response against vitiligo-associated autoantigens, including MART-1, tyrosinase, and gp100 (2-11).

The epidemiology of this disease indicates that the prevalence of vitiligo ranges from 0.5 to $1 \%$ of the general population, and the disease is equally distributed in females and males (12). Considering that vitiligo is a common disease associated with various autoimmune diseases and that these comorbidities are usually evaluated as separate clinical entities, it is of interest to define the rheumatic comorbidities that can be associated with vitiligo as part of a multi-autoimmune syndrome, wherein this designation applies to the association of three or more autoimmune diseases in the same patient.

The aim of the present study was to determine the association of vitiligo with multiple autoimmune rheumatic diseases in a database covering a period of 10 years.

\section{Materials and methods}

Subjects. The clinical files of patients who were treated in the Department of Rheumatology from 2005 and 2015 were reviewed. Using the data files, the main disease that led to the evaluation was identified. Subsequently, the number of patients with or 
without rheumatic autoimmune diseases such as osteoarthritis, lumbalgia or tendinitis associated with vitiligo was determined. Each patient was reviewed by a skilled rheumatologist and dermatologist, respectively, and clinical criteria for disease for classification in each autoimmune disease was followed. Additionally, in each patient with vitiligo, the availability of serum samples stored in a freezer was determined, allowing for evaluation of the anti-melanocyte and anti-tyrosinase antibodies, which are markers of autoimmune vitiligo. Additionally, a group of rheumatic patients lacking autoimmune disease was included as the control group. Autoimmune co-morbidities and autoantibodies were assessed in the two groups.

The study was approved by the ethics committee of Universidad Autónoma de Zacatecas (Zacatecas, Mexico). Patient consent was obtained from each patient.

Anti-melanocyte antibodies. An intradermal nevus, $\sim 0.5 \mathrm{~cm}$ in diameter, of the preauricular region in a patient who was 27 years with no clinical or serological manifestations of autoimmunity was removed for cosmetic reasons. Prior to this procedure, a signed authorization was obtained to use part of the nevus as an antigenic source. The lesion was divided into two parts; one part was sent for histopathological analysis and another part was used for indirect immunofluorescence studies (13). The tissue was transported to the laboratory on ice, and was subsequently embedded in Tissue-Tek OCT ${ }^{\circledR}$ (Leica Biosystems, Nussloch, Germany) and frozen at $-20^{\circ} \mathrm{C}$. Tissue samples were cut at $4 \mu \mathrm{m}$ using a cryostat $\left(-20^{\circ} \mathrm{C}\right)$, and the slices were fixed on slides. The serum samples were tested at dilutions of 1:20 to 1:160, incubated with the antigenic source for $30 \mathrm{~min}$ in a moist chamber, and the slides were washed three times in phosphate-buffered saline (PBS) ( $\mathrm{pH}$ 7.2). This step was followed by a 30-min incubation with polyvalent FITC-labelled rabbit anti-human antiserum (cat. no. F4637; 1:80; Sigma-Aldrich, St. Louis, MO, USA). After three additional PBS washes, the slides were mounted with glycerol-PBS (9:1), and two independent observers evaluated the samples using an Olympus B-Max BX-40 fluorescence microscope (Olympus, Tokyo, Japan) in a blinded manner.

Anti-tyrosinase antibodies. The specificity of the sera to tyrosinase was evaluated in triplicate by ELISA as previously described (14) with modifications. Polystyrene plates were covered overnight with $10 \mu \mathrm{g}$ of tyrosinase fragment 369-377 (cat. no. T8455; Sigma-Aldrich) dissolved in $70 \%$ ethanol. Uncovered sites were neutralized with $4 \%$ BSA. The serum samples were diluted 1:100 and applied into the microwells. They were then incubated for $2 \mathrm{~h}$ at room temperature. After 3 washes, the samples were incubated with secondary antibody goat anti-human IgG HRP (cat. no. sc-2453; Santa Cruz Biotechnology, Inc., Santa Cruz, CA, USA) for $1 \mathrm{~h}$. After washing again, the colour reaction was developed with TMB in a $30-\mathrm{min}$ incubation. The reaction was then stopped with $0.5 \mathrm{M}$ sulfuric acid and the microplates were read at $450 \mathrm{~nm}$ using a microplate reader (Thermo Fisher Scientific, Inc., Shanghai, China).

Other autoantibodies. Anti-thyroid antibodies on a commercial antigenic source, anti-nuclear antibodies in HEp-2 cells (Immuno Concepts, Inc., Sacramento, CA, USA) and anti-epithelial antibodies, using as antigen source cow nose, were evaluated with indirect immunofluorescence.
Statistical analysis. The probability of the association between vitiligo and certain autoimmune rheumatic disease was calculated using the $\chi^{2}$ test. The relative risk and odds ratios (OR), with $95 \%$ of confidence intervals, were determined using the Prism software program (GraphPad Software, Inc., San Diego, CA, USA). $\mathrm{P}<0.0001$ was considered statistically significant.

\section{Results}

Clinical records. In 10 years, a total of 5,251 records were filed. Each record corresponded to one patient, and these patients were evaluated over multiple rheumatology consultations. From these, 2,824 files corresponded to patients with autoimmune disease (54\%), while the remaining 2,427 (46\%) had other rheumatic diseases, including degenerative, metabolic or other diseases. The most prevalent autoimmune disease was exhibited by rheumatoid arthritis (RA) patients who met the ACR/EULAR classification criteria (15), which accounted for $43 \%$ of autoimmune rheumatic diseases. This was followed by $26 \%$ with lupus erythematosus, which met the ACR revised criteria for SLE (16). Other diseases, such as scleroderma, accounted for a lower proportion of the cases $(2.4 \%)$. Of the total number of autoimmune rheumatic diseases, 19 patients, 16 women and 3 men, with a mean age of 36 years, had GV (17), corresponding to $0.672 \%$ of the sample. Clinically, most of the patients had skin depigmentation distributed along the face, hands and trunk (Fig. 1). In contrast to the control group, only one patient with osteoarthritis had localized spots of vitiligo, and the prevalence was $0.0412 \%$. The differences between the two groups were significant $(\mathrm{P}<0.0002)$ (Fig. 1). Of note, the relative risk for developing vitiligo was significantly increased in patients who had an additional autoimmune comorbidity, such as celiac of $33 \%$ (18) or thyroid disease in 19\% (19) (Table I).

Autoantibodies. Ninety-two percent of the group with autoimmune rheumatic disease and associated vitiligo tested positive for anti-melanocyte antibodies. Therefore, fluorescence was observed in scattered melanocytes along the epidermis as well as in melanosomes and melanin granules for some patients. The antibody titres varied across the patients. By contrast, the anti-tyrosinase antibodies were positive in $84 \%$ of the patients (Fig. 2).

Other shared autoantibodies included rheumatoid factor and anti-CCP, ANA, anti-thyroid and anti-epithelial antibodies although none of the patients had symptoms of pemphigus.

\section{Discussion}

Vitiligo is a disease characterized by the loss of pigmentproducing cells. The disease has psychological and social consequences, and in some cases it is caused by autoimmunity. The aim of the present study was to determine the prevalence of vitiligo associated with other autoimmune rheumatic diseases. The main results of the present study were as follows. First, the prevalence of vitiligo associated with autoimmune rheumatic disease was 0.672 . Second, autoantibodies against vitiligo-associated antigens were present in the $92 \%$ of the autoimmune group. Third, RA and lupus were frequently associated with vitiligo, although the relative risk of vitiligo 
A Patients consulted in 10 years

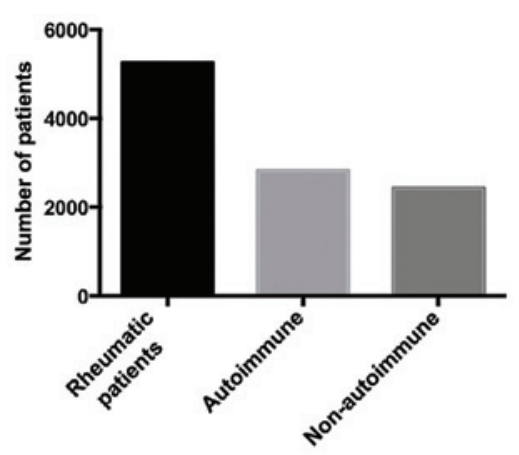

C

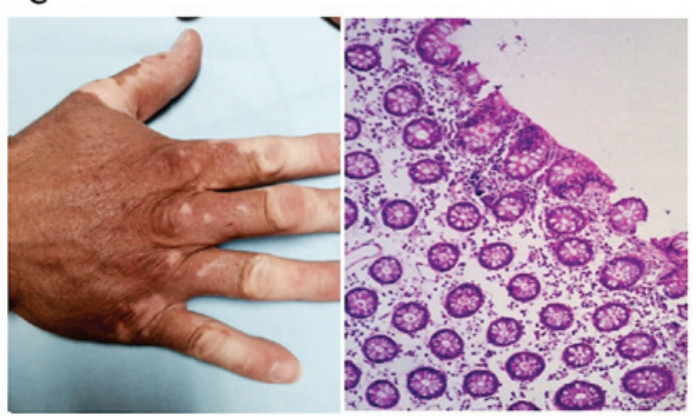

B Vitiligo comorbidities
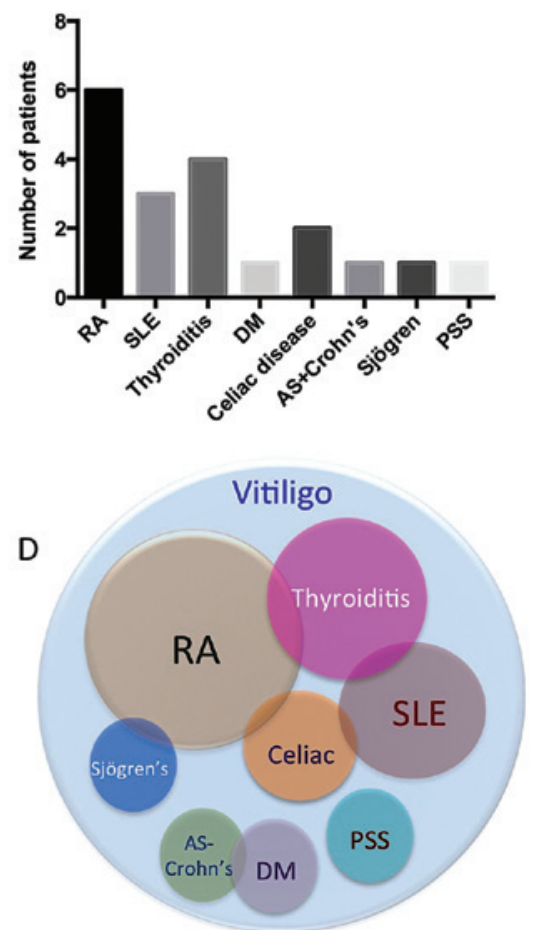

Figure 1. The vitiligo prevalence in rheumatic diseases. (A) Total number of patients grouped into autoimmune and non-autoimmune rheumatic diseases. (B) Patients with autoimmune rheumatic diseases associated with vitiligo. (C) Hand from a patient with dermatomyositis, vitiligo and Crohn's disease (as shown in the intestinal histology). (D) Drawing shows the clinical overlap of vitiligo and the comorbidities in the present study. RA, rheumatoid arthritis; SLE, systemic lupus erythematosus; PSS, progressive systemic sclerosis; AS, ankylosing spondylitis; DM, dermatomyositis.

Table I. Relative risk for developing vitiligo.

\begin{tabular}{lcccccc}
\hline Disease & No. of patients & Vitiligo & Prevalence & P-value & Relative risk & OR \\
\hline NARD & 2,427 & 1 & 0.041 & & & \\
ARD & 2,829 & 19 & 0.672 & 0.0002 & 0.0612 & 0.0608 \\
RA & 1,899 & 6 & 0.315 & 0.0257 & 0.1304 & 0.1300 \\
SLE & 742 & 3 & 0.404 & 0.0148 & 0.1019 & 0.1015 \\
Sjögren & 91 & 1 & 1.098 & 0.0004 & 0.0374 & 0.0371 \\
PSS & 68 & 1 & 1.470 & 0.0001 & 0.0280 & 0.0276 \\
DM & 20 & 1 & 5.000 & 0.0001 & 0.0082 & 0.0078 \\
Tyroiditis + RA or SLE & 21 & 4 & 19.047 & 0.0001 & 0.0021 & $0.0017^{\mathrm{a}}$ \\
Celiac disease + SLE or RA & 6 & 2 & 33.333 & 0.0001 & 0.0012 & $0.0008^{\mathrm{a}}$ \\
AS + Crohn's disease & 4 & 1 & 25.000 & 0.0001 & 0.0016 & $0.0012^{\mathrm{a}}$ \\
\hline
\end{tabular}

OR, odds ratio; NARD, non-autoimmune rheumatic disease; ARD, autoimmune rheumatic disease; RA, rheumatoid arthritis; SLE, systemic lupus erythematosus; AS, ankylosing spondylitis. ${ }^{a}$ Highly significant, $\mathrm{P}<0.0001$.

as an event occurring, measured the magnitude of association using the cumulative incidence, rather than the total number. This measure showed the relative risk of vitiligo in RA was not different than the prevalence reported in the general population. Notably, the vitiligo risk was markedly increased when the two diseases were associated with thyroid or celiac disease.

Vitiligo has been extensively studied. In 1979, Goudie et al (20) brought attention to the comorbidities of vitiligo, suggesting that this disease constituted a set of mosaic patches with distinctive shared characteristics of autoimmunity. Traditionally, vitiligo alone has been extensively studied worldwide, including in Latin America. The present study was performed in the mestizo population of a central region of Mexico. The mestizo population results from a mixture between Amerindian Caucasian and African genes (21). In Mexico, the prevalence of vitiligo in children has been estimated as $2.6 \%$ (22). The present study addresses the prevalence of vitiligo in adult patients with rheumatic disease, and the global prevalence of vitiligo detected in the present study was 0.380 , which is lower than 

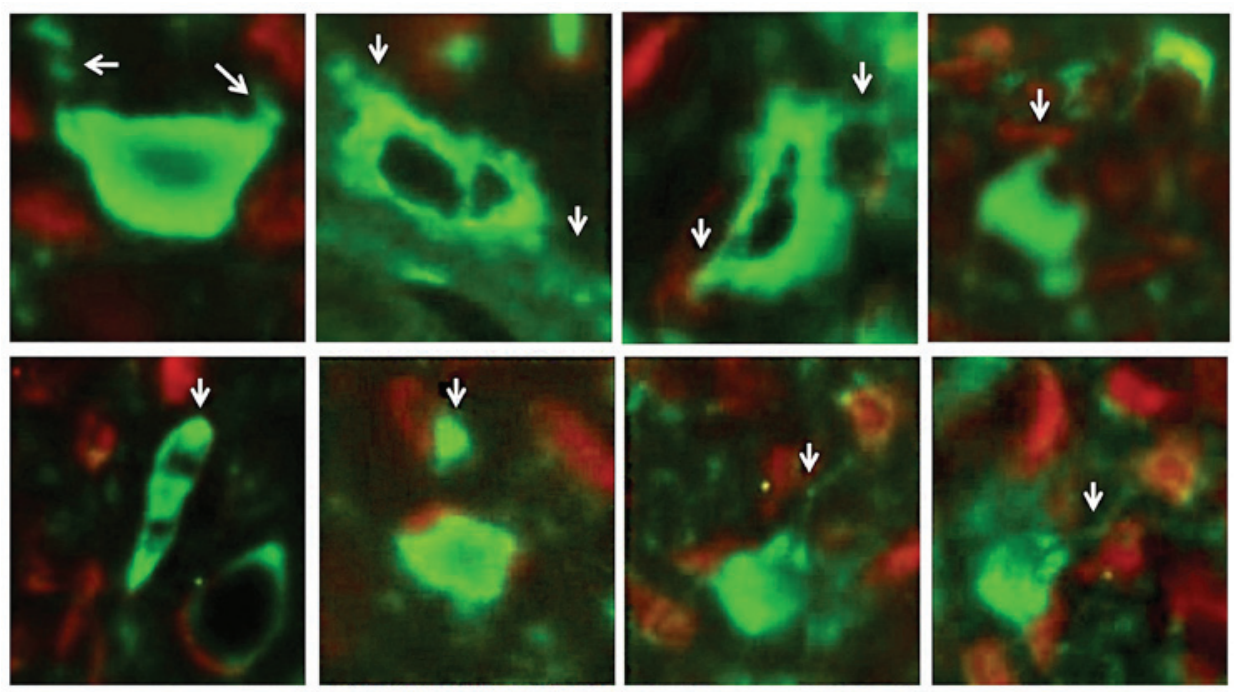

Autoantibodies in vitiligo
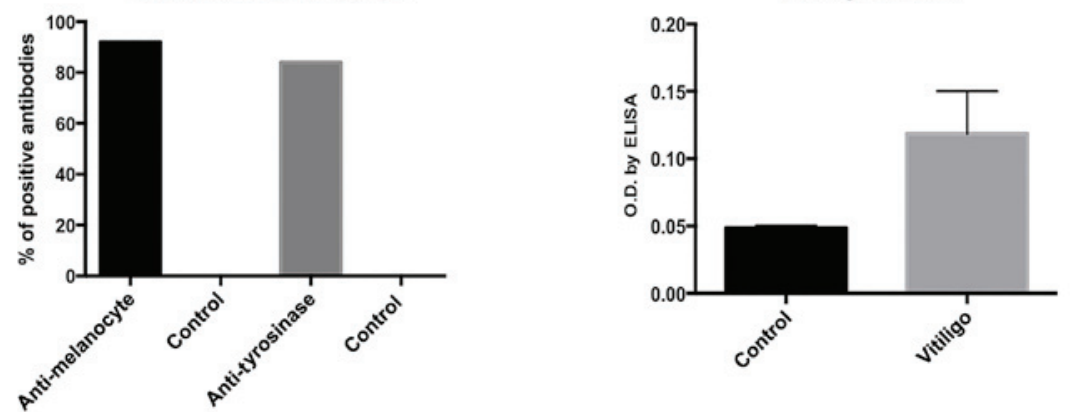

Figure 2. Antibodies against vitiligo autoantigens. Superior panel, representative immunofluorescence antibodies against melanocytes. Inferior panel (left), a graph with the percentage of positivity of vitiligo autoantoibodies. Inferior panel (right), the ELISA graph for anti-thyrosinase antibodies.

the $0.5-2 \%$ reported in the overall worldwide population by Krüger and Schallreuter (23). The differences with the present results can be explained by ethnic factors or different methodologies used in the patients included in each study. Of note, the prevalence of vitiligo is variable; for instance, the prevalence in the Korean population is $0.12 \%$ (24), while it is lower $(0.093 \%)$ in a Chinese Shaanxi Province (25). In both instances, the prevalence in some Asiatic countries is lower than in the present study.

Concurrence of various autoimmune diseases in a single patient captured the attention of different clinical research groups, and diverse names have been given to these associated comorbidities, including polyautoimmunity, multiple autoimmunity, and shared autoimmunity (26-30). The autoimmune rheumatic comorbidities associated with vitiligo are variable, including RA and psoriasis (31-33). In the Middle Eastern population, the association of vitiligo with RA is related to the $\mathrm{TNF} \alpha-308 \mathrm{~A} / \mathrm{G}$ promoter (34), which probably reflects that this cytokine participates in melanocyte destruction. Sjögren disease has been reported to be associated with vitiligo (35). Other autoimmune comorbidities that accompany rheumatic diseases and vitiligo include pernicious anemia (36), Graves' disease, autoimmune haemolytic anemia (37), thyroid and celiac disease and other pathologies (38-42). Common pathogenic mechanisms that are suggested to facilitate these comorbidities involve the protein tyrosine phosphatase non-receptor type 22 (PTPN22) gene, which seems to increase the susceptibility for one patient to have a cluster of different autoimmune diseases. This cluster includes type I diabetes, RA, autoimmune thrombocytopenia, inflammatory myopathies, Graves' disease, SLE, ANCA-associated vasculitis, and Cohn's disease (43). On the other hand, HLA-DQ pleiotropic genes are also involved in the pathogenesis of multiple autoimmunity (44).

In conclusion, based on the results of this study, the association between vitiligo and one autoimmune rheumatic disease is relatively low, which is probably similar to the prevalence of vitiligo in the general population. Nevertheless, when this disease is associated with more than two autoimmune comorbidities, including thyroid or celiac disease, its relative risk is notably increased. In such a case, a multiple autoimmune syndrome should be suspected.

\section{References}

1. Spritz RA: The genetics of vitiligo. J Invest Dermatol 131 (E1): E18-E20, 2011.

2. Birlea SA, Jin Y, Bennett DC, Herbstman DM, Wallace MR, McCormack WT, Kemp EH, Gawkrodger DJ, Weetman AP, Picardo M, et al: Comprehensive association analysis of candidate genes for generalized vitiligo supports XBP1, FOXP3, and TSLP. J Invest Dermatol 131: 371-381, 2011.

3. Quan C, Ren YQ, Xiang LH, Sun LD, Xu AE, Gao XH, Chen HD, $\mathrm{Pu} \mathrm{XM}, \mathrm{Wu} \mathrm{RN}$, Liang CZ, et al: Genome-wide association study for vitiligo identifies susceptibility loci at 6q27 and the MHC. Nat Genet 42: 614-618, 2010.

4. Rahoma SF, Sandhu HK, McDonagh AJ, Gawkrodger DJ, Weetman AP and Kemp EH: Epitopes, avidity and IgG subclasses of tyrosine hydroxylase autoantibodies in vitiligo and alopecia areata patients. Br J Dermatol 167: 17-28, 2012. 
5. Park YK, Kim NS, Hann SK and Im S: Identification of autoantibody to melanocytes and characterization of vitiligo antigen in vitiligo patients. J Dermatol Sci 11: 111-120, 1996.

6. Li S, Yao W, Pan Q, Tang X, Zhao S, Wang W, Zhu Z, Gao J, Sheng Y,Zhou F, et al: Association analysis revealed one susceptibility locus for vitiligo with immune-related diseases in the Chinese Han population. Immunogenetics 67: 347-354, 2015.

7. Evoli A, Caliandro P, Iorio R, Alboini PE, Damato V, LaTorre G, Provenzano C, Marino M, Lauriola L, Scuderi F, et al: Poly-autoimmunity in patients with myasthenia gravis: A single-center experience. Autoimmunity 48: 412-417, 2015.

8. Byrne KT, Zhang P, Steinberg SM and Turk MJ: Autoimmune vitiligo does not require the ongoing priming of naive $\mathrm{CD} 8$ $\mathrm{T}$ cells for disease progression or associated protection against melanoma. J Immunol 192: 1433-1439, 2014.

9. Colucci R, Böhm M and Moretti S: Commentary from the Editorial Board to Vitiligo: Interplay between oxidative stress and immune system (Laddha et al). Exp Dermatol 22: 397-398, 2013.

10. Ingordo V, Cazzaniga S, Raone B, Digiuseppe MD Musumeci ML, Fai D, Pellegrino M, Pezzarossa E, Di Lernia V, Battarra VC, et al: Circulating autoantibodies and autoimmune comorbidities in vitiligo patients: a multicenter Italian study. Dermatology 228: 240-249, 2014.

11. Gregg RK, Nichols L, Chen Y, Lu B and Engelhard VH: Mechanisms of spatial and temporal development of autoimmune vitiligo in tyrosinase-specific TCR transgenic mice. J Immunol 184: 1909-1917, 2010.

12. Alkhateeb A, Fain PR, Thody A, Bennett DC and Spritz RA Epidemiology of vitiligo and associated autoimmune diseases in Caucasian probands and their families. Pigment Cell Res 16 208-214, 2003.

13. Petersen M, Davids LM and Kidson SH: Simultaneous immunofluorescent labeling using anti-BrdU monoclonal antibody and a melanocyte-specific marker in formalin-fixed paraffin-embedded human skin samples. Appl Immunohistochem Mol Morphol 20 : 614-617, 2012.

14. Okamoto T, Irie RF, Fujii S, Huang SK, Nizze AJ, Morton DL and Hoon DS: Anti-tyrosinase-related protein-2 immune response in vitiligo patients and melanoma patients receiving active-specific immunotherapy. J Invest Dermatol 111: 1034-1039, 1998.

15. Aletaha D, Neogi T, Silman AJ, Funovits J, Felson DT, Bingham CO III, Birnbaum NS, Burmester GR, Bykerk VP, Cohen MD, et al: 2010 Rheumatoid arthritis classification criteria: an American College of Rheumatology/European League Against Rheumatism collaborative initiative. Arthritis Rheum 62: 2569-2581, 2010.

16. Hochberg MC: Updating the American College of Rheumatology revised criteria for the classification of systemic lupus erythematosus. Arthritis Rheum 40: 1725, 1997.

17. Ezzedine K, Lim HW, Suzuki T, Katayama I, Hamzavi I, Lan CC, Goh BK, Anbar T, Silva de Castro C, Lee AY, et al; Vitiligo Global Issue Consensus Conference Panelists: Revised classification/nomenclature of vitiligo and related issues: The Vitiligo Global Issues Consensus Conference. Pigment Cell Melanoma Res 25: E1-E13, 2012

18. Rubio-Tapia A, Hill ID, Kelly CP, Calderwood AH and Murray JA; American College of Gastroenterology: ACG clinical guidelines: diagnosis and management of celiac disease. Am J Gastroenterol 108: 656-676, quiz 677, 2013.

19. Garber JR, Cobin RH, Gharib H, Hennessey JV, Klein I, Mechanick JI, Pessah-Pollack R, Singer PA and Woeber KA; American Association of Clinical Endocrinologists and American Thyroid Association Taskforce on Hypothyroidism in Adults: Clinical practice guidelines for hypothyroidism in adults: cosponsored by the American Association of Clinical Endocrinologists and the American Thyroid Association. Endocr Pract 18: 988-1028, 2012

20. Goudie RB, Spence JC and MacKie R: Vitiligo patterns simulating autoimmune and rheumatic diseases. Lancet 2: 393-395, 1979 .

21. Santana C, Noris G, Meraz-Ríos MA, Magaña JJ, Calderon-Aranda ES, Muñoz ML and Gómez R: Genetic analysis of 17 Y-STRs in a Mestizo population from the Central Valley of Mexico. Hum Biol 86: 289-312, 2014.

22. Ruiz-Maldonado R, Tamayo Sánchez L and Velázquez E: Epidemiology of skin diseases in 10,000 patients of pediatric age. Bol Med Hosp Infant Mex 34: 137-161, 1977 (In Spanish).
23. Krüger $\mathrm{C}$ and Schallreuter KU: A review of the worldwide prevalence of vitiligo in children/adolescents and adults. Int J Dermatol 51: 1206-1212, 2012.

24. Lee H, Lee MH, Lee DY, Kang HY, Kim KH, Choi GS, Shin J, Lee HJ, Kim DH, Kim TH, et al: Prevalence of vitiligo and associated comorbidities in Korea. Yonsei Med J 56: 719-725, 2015.

25. Lu T, Gao T, Wang A, Jin Y, Li Q and Li C: Vitiligo prevalence study in Shaanxi Province, China. Int J Dermatol 46: 47-51, 2007.

26. Humbert P and Dupond JL: Multiple autoimmune syndromes. Ann Med Interne (Paris) 139: 159-168, 1988 (In French).

27. Anaya JM: The diagnosis and clinical significance of polyautoimmunity. Autoimmun Rev 13: 423-426, 2014.

28. Anaya JM, Castiblanco J, Rojas-Villarraga A, Pineda-Tamayo R, Levy RA, Gómez-Puerta J, Dias C, Mantilla RD, Gallo JE, Cervera $\mathrm{R}$, et al: The multiple autoimmune syndromes. A clue for the autoimmune tautology. Clin Rev Allergy Immunol 43: 256-264, 2012

29. Rojas-Villarraga A, Amaya-Amaya J, Rodriguez-Rodriguez A, Mantilla RD and Anaya JM: Introducing polyautoimmunity: secondary autoimmune diseases no longer exist. Autoimmune Dis 2012: 254319, 2012

30. Cojocaru M, Cojocaru IM and Silosi I: Multiple autoimmune syndrome. Maedica (Buchar) 5: 132-134, 2010.

31. Liu JB, Li M, Yang S, Gui JP, Wang HY, Du WH, Zhao XY, Ren YQ, Zhu YG and Zhang XJ: Clinical profiles of vitiligo in China: An analysis of 3742 patients. Clin Exp Dermatol 30: 327-331, 2005.

32. Zhang Z, Xu SX, Zhang FY, Yin XY, Yang S, Xiao FL, Du WH, Wang JF, Lv YM, Tang HY, et al: The analysis of genetics and associated autoimmune diseases in Chinese vitiligo patients. Arch Dermatol Res 301: 167-173, 2009.

33. Laberge G, Mailloux CM, Gowan K, Holland P, Bennett DC, Fain PR and Spritz RA: Early disease onset and increased risk of other autoimmune diseases in familial generalized vitiligo. Pigment Cell Res 18: 300-305, 2005.

34. Lee YH and Bae SC: Associations between TNF- $\alpha$ polymorphisms and susceptibility to rheumatoid arthritis and vitiligo: A meta-analysis. Genet Mol Res 14: 5548-5559, 2015

35. Roguedas AM, Misery L, Sassolas B, Le Masson G, Pennec YL and Youinou P: Cutaneous manifestations of primary Sjögren's syndrome are underestimated. Clin Exp Rheumatol 22: 632-636, 2004.

36. Abraham Z, Rozenbaum M, Glück Z, Feuerman EJ, Lahat N and Kinarty A: Vitiligo, rheumatoid arthritis and pernicious anemia. J Dermatol 20: 418-423, 1993.

37. Chan HL, Lee YS, Hong HS and Kuo TT: Anticentromere antibodies (ACA): Clinical distribution and disease specificity. Clin Exp Dermatol 19: 298-302, 1994.

38. Ponto KA, Schuppan D, Zwiener I, Binder H, Mirshahi A, Diana T, Pitz S, Pfeiffer N and Kahaly GJ: Thyroid-associated orbitopathy is linked to gastrointestinal autoimmunity. Clin Exp Immunol 178: 57-64, 2014.

39. Boelaert K, Newby PR, Simmonds MJ, Holder RL, Carr-Smith JD, Heward JM, Manji N, Allahabadia A, Armitage M, Chatterjee KV, et al: Prevalence and relative risk of other autoimmune diseases in subjects with autoimmune thyroid disease. Am J Med 123: 183.e1-183.e9, 2010.

40. Amador-Patarroyo MJ, Arbelaez JG, Mantilla RD, Rodriguez-Rodriguez A, Cárdenas-Roldán J, Pineda-Tamayo R, Guarin MR, Kleine LL, Rojas-Villarraga A and Anaya JM: Sjögren's syndrome at the crossroad of polyautoimmunity. J Autoimmun 39: 199-205, 2012

41. Larizza D, Calcaterra V, Klersy C, Badulli C, Caramagna C, Ricci A, Brambilla P, Salvaneschi L and Martinetti M: Common immunogenetic profile in children with multiple autoimmune diseases: the signature of HLA-DQ pleiotropic genes. Autoimmunity 45: 470-475, 2012.

42. Zakka LR, Reche PA and Ahmed AR: The molecular basis for the presence of two autoimmune diseases occurring simultaneously - preliminary observations based on computer analysis. Autoimmunity 45: 253-263, 2012.

43. Zheng J, Ibrahim S, Petersen F and Yu X: Meta-analysis reveals an association of PTPN22 C1858T with autoimmune diseases, which depends on the localization of the affected tissue. Genes Immun 13: 641-652, 2012.

44. Cárdenas-Roldán J, Rojas-Villarraga A and Anaya JM: How do autoimmune diseases cluster in families? A systematic review and meta-analysis. BMC Med 11: 73, 2013. 